\title{
Guiding an HPSG Parser using Semantic and Pragmatic Expectations
}

\author{
Jim Skon \\ Computer and Information Science Department \\ The Ohio State University \\ Columbus, OH 43210, USA \\ Internet: skon@cis.ohio-state.edu
}

\begin{abstract}
1
Efficient natural language generation has been successfully demonstrated using highly compiled knowledge about speech acts and their related social actions. A design and prototype implementation of a parser which utilizes this same pragmatic knowledge to efficiently guide parsing is presented. Such guidance is shown to prune the search space and thus avoid needless processing of pragmatically unlikely constituent structures.
\end{abstract}

\section{INTRODUCTION}

The use of purely syntactic knowledge during the parse phase of natural language understanding yields considerable local ambiguity (consideration of impossible subconstituents) as well global ambiguity (construction of syntactically valid parses not applicable to the socio-pragmatic context).

This research investigates bringing socio-pragmatic knowledge to bear during the parse, while maintaining a domain independent grammar and parser. The particular technique explored uses knowledge about the pragmatic context to order the consideration of proposed parse constituents, thus guiding the parser to consider the best (wrt the expectations) solutions first. Such a search may be classified as a bestfirst search.

The theoretical models used to represent the pragmatic knowledge in this study are based on Halliday's Systemic Grammar and a model of the pragmatics of conversation. The model used to represent the syntax and domain independent semantic knowledge is HPSG - Head-driven Phrase Structure Grammar.

\section{BACKGROUND}

Patten, Geis and Becker (1992) demonstrate the application of knowledge compilation to achieve the rapid generation of natural language. Their mechanism is based on Halliday's systemic networks, and on Geis' theory of the pragmatics of conversation. A model of conversation using principled compilation of pragmatic knowledge and other linguistic knowledge is used to permit the application of pragmatic inference without expensive computation. A pragmatic component is used to model social action, including speech acts, and utilize conventions of ușe involving such features of context such as politeness, register, and stylistic features. These politeness features are critical to the account of indirect speech acts. This pragmatic knowledge is compiled into course-grained knowledge in the form of a classification hierarchy. A planner component uses knowledge about conditions which need to be satisfied (discourse goals) to produce a set of pragmatic features which characterize a desired utterance. These features are mapped into the systemic

1 Research Funded by The Ohio State Center for Cognitlve Science and The Ohio State Departments of Computer and Information Science and Linguistics grammar (using compiled knowledge) which is then used to realize the actual utterance.

The syntactic/semantic component used in this study is a parser based on the HPSG (Head Driven Phrase Structure Grammar) theory of grammar (Pollard and Sag, 1992). HPSG models all linguistic constituents in terms of partial information structures called feature structures. Linguistic signs incorporate simultaneous representation of phonological, syntactic, and semantic attributes of grammatical constituents. HPSG is a lexicalized theory, with the lexical definitions, rather then phrase structure rules, specifying most configurational constraints. Control (such as subcategorization, for example) is asserted by the use of HPSG constraints - partially filled in feature structures called feature descriptions, which constrain possible HPSG feature structures by asserting specific attributes and/or labels.

A HPSG based chart parser, under development at the author's university, was used for the implementation part of this study.

\section{FEATURE MAPPING}

Planning \& generation of coherent "speech" in a conversation requires some understanding of the "hearer's" perspective. Thus the speaker naturally has some (limited) knowledge about possible responses from the hearer. This knowledge can be given to the same planner used for generation, producing a partial set of pragmatic features or expectations. These pragmatic expectations can then be mapped into the systemic grammar, producing a set of semantic and syntactic expectations about what other participants in the conversation will say.

The technique explored here is to bring such expectations to bear during the parse process, guiding the parser to the most likely solution in a best-first manner. It is thus necessary that the generated expectations be mapped into a form which can be directly compared with constituents proposed within the HPSG parse.

Consider the sentence "Robin promised to come at noon", with the following context:

Sandy: "I guess we should get started, what time did they say they would be here?"

Kim: "Robin promised to come at noon"

A set of plausible partial expectations generated by the pragmatic and systemic components in anticipation of Kim's response might be:

$$
\begin{aligned}
& \text { ((S) (UNMARKED-DECLARATIVE)) } \\
& \text { ((S SUBJECT) (PROPER)) } \\
& \text { ((S BETA) (NONFINITEPRED)) } \\
& \text { ((S PREDICATOR) (PROMISED)) } \\
& \text { ((S BETA TEMPORAL) (PP)) } \\
& \text { ((S BETA PREDICATOR) (ARRIVAL)) }
\end{aligned}
$$

In these expectations the first list of each pair (e.g. (S BETA)) represents a functional role within the expected sentence. The 
second list in each pair are sets (in this case singleton) of expected features for the associated functional roles. These expected features assert expectations which are both semantic (e.g. PROMISED) and syntactic (e.g. ((S BETA TEMPORAL) (PP)) asserts both the existance and location of a temporal adjunct PP).

Note that in these expectations the temporal adjunct "at noon" should modify the embedded clause "to come", as would be expected in the specified context.

Next consider the possible HPSG parses of the example sentence. Figures 1 and 2 below illustrate two semantically distinct parses generated by our HPSG parser.

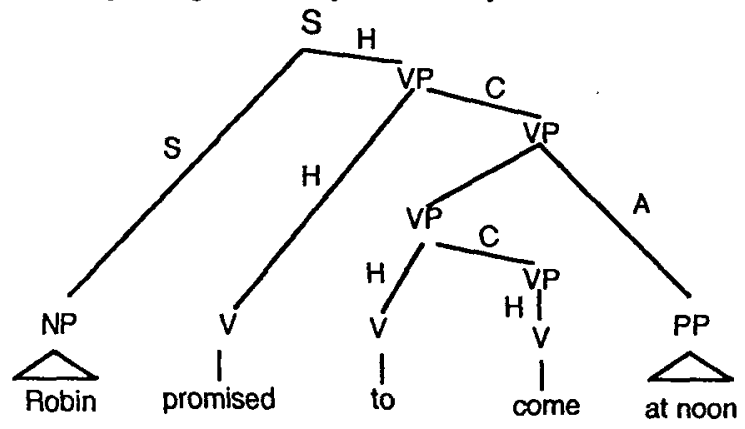

Figure 1.

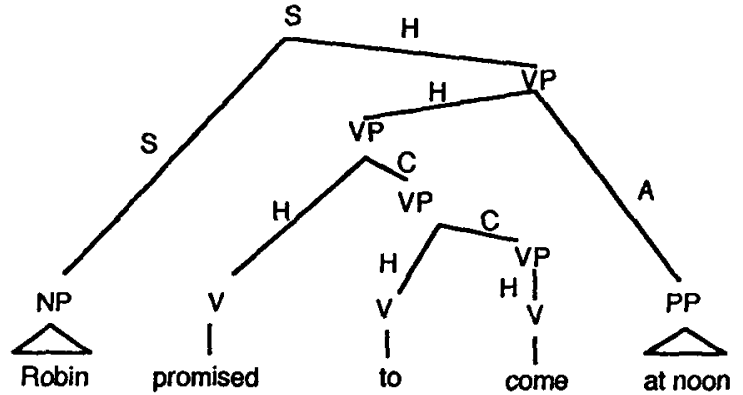

Figure 2.

Mapping expected features into HPSG constraints: Features generated from pragmatic expectations can be mapped into constraints on HPSG structures, stated in terms of feature descriptions. Below are the HPSG feature descriptions corresponding to the pragmatically generated features PP and UNMARKED-DECLARATIVE.

$$
\left.\begin{array}{l}
\mathrm{PP}=[\text { SYNSEMILOCICAT } \\
\text { phrase }
\end{array}\left[\begin{array}{l}
\text { HEAD prep } \\
\text { MARKING unmarked }
\end{array}\right]\right]
$$

Figure 3.

$$
\begin{aligned}
& \text { UNMARKED-DECLARATIVE }= \\
& \left.\qquad \begin{array}{l}
\text { DTRSIHEAD-DTRISYNSEM vp } \\
\text { SUBJ-DTRISYNSEMILOCICATIHEAD noun }
\end{array}\right]
\end{aligned}
$$

Figure 4.

\author{
Mapping expected functional roles into HPSG \\ constituent structure: \\ Pragmatic expectations are expected within certain \\ functional roles, such a SUBJECT, PREDICATOR, BETA
} (the embedded clause) etc. This structural information must be used to assert the constraints into the relevant HPSG substructures. This mapping is not as straightforward as the feature mapping technique, as the structure induced by the systemic grammar is "flatter" than the structure produced by HPSG.

Consider the following pragmatically generated expectation:

((S TEMPORAL) (PP))

Such an expectation may be realized by great variety of HPSG structural realizations. e.g.:

1. Kim ran at noon

2. Kim could run home at noon

3. Kim could have been running home at noon

4. Kim ran east at noon.

In these examples modal verb operators (1-3) and multiple adjuncts (4) vary the actual structural depth of the temporal PP within the HPSG model. Thus a given systemic role path may have numerous HPSG constituent path realizations. One possible mapping technique is to generate constraints expressing all possible HPSG structural variants. This, however would lead in many cases to a combinatorial explosion of constraints. The technique employed by this study was to add a new clause attribute to verbal HPSG signs, and use this attribute to embed within the signs a "clausally flattened" structures. Each HPSG verbal sign in the same clause structure shares the same clausal value. The clause value is a structure with labels for each systemic role, where each label points to the constituent which fills that role in the given verbal clause. A clausal boundry is said to exist between distinct clausal domains. A clausal structure is illustrated in figure 5 :

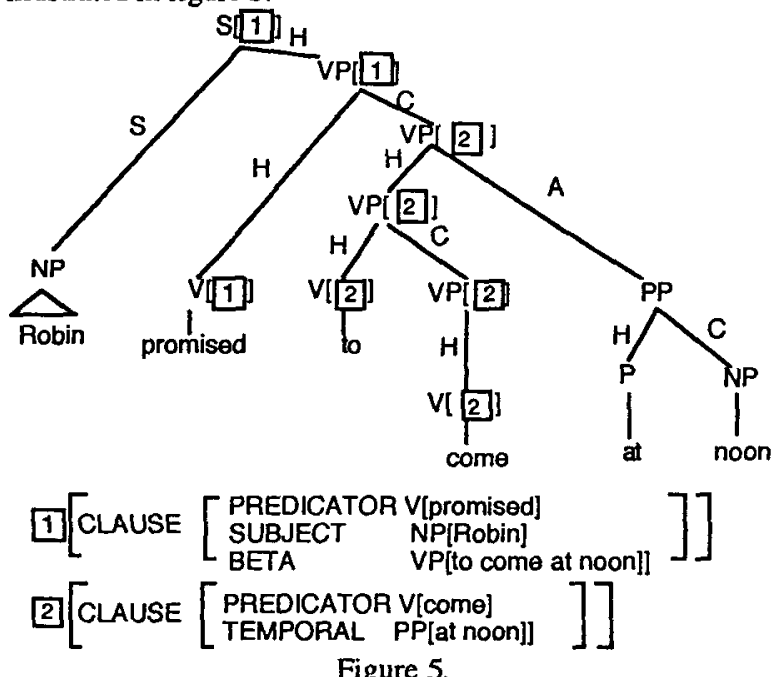

Figure 5.

The current mapping only considers the mapping of roles within verbal signs. Similar role structures may exist for other constituent types, such as for noun phrase. Thus far the verbal clause boundary definition has been adequate for other phrasal structures.

\section{GUIDING THE HPSG PARSE}

The guidance strategy employed is to evaluate all proposed edges (i.e. complete and partially complete constituents) against the expectations, ranking each based on the relative similarity with the expectations. These edges are 
then placed in an agenda (a list of priority queues) and removed from the agenda and included in the partial parse in a best first order.

Critical to the success of a best-first algorithm is the heuristic evaluation function used to order the proposed constituents.

The heuristic evaluation function:

The heuristic evaluation function is based on three specific types of tests:

1. Role match - does a constituent match a role's set of expected features?

II. Role path match - is a constituent role path compatible with the roles of its children?

III. Clausal completeness - are all clausal roles expected for this constituent present?

Tests II and III above require that constituents under consideration have roles already assigned to them. For example, in the case of II, the test requires roles for both the new constituent and the proposed daughters of the constituent. But since the parse strategy employeed is bottom-up, role paths cannot be anchored to a root, and thus fully known, until parse completion. The solution to this dilemma is to hypothesise a constituent's role using a process similar to abduction. Two types of knowledge are exploited in this process. First, roles with features which subsume or are consistant with a proposed constituent are considered good candidate roles. Also, roles may also be inferred by projecting up from the roles already hypothesized for the children. By intersecting these two sources of role evidence, the list of hypothesized roles can be refined (by ruling out roles without both types of evidence). In this manner the hypothesized roles of later constituents can be refined from descendant constituents. In the case of roles projected from daughters, clausal boundary knowledge must be applied to correctly infer the parent role.

\section{EVALUATION \& TESTING}

The techniques described here have been used successfully to guide the parsing of several sentences taken from real conversations. The pragmatic and semantic knowledge already existed from Patten's research (Patten, 1992) to generate these sentences. A subset of this knowledge, judged to represent the partial knowledge available to a listener, was used to generate expectations in the form described above.

The parser used in this study by default produced all possible parses. The modified version attempts to converge on the "expected" parse first, and terminate. For each sentence tested the parser converges on the correct parse first. When the expectations are modified to expect a different parse, a different (and correct) parse is found first. The results in terms of speedup vary considerably depending on the level of ambiguity present in the sentence. The most complex sentence parsed thus far exhibits considerable speedup. When unguided, the parser produces 24 parses, and considers a total of 252 distinct constituents. In the guided case, the parser only considers 39 constituents, and converges on the one "correct" parse first. Within the current testing environment, this guidence results in a greater then ten-fold speedup in terms of CPU time.

\section{SUMMARY}

Pragmatic knowledge about language usage in routine conversational contexts can be highly compiled. This knowledge can be used to produce semantic and syntactic expectations about next turns in conversation, especially of next turns that are second members of adjacency pairs (Schegloff \& Sacks 1973). By mapping expected features into HPSG constraints, and by augmenting HPSG sign structures to model the role structure of systemic grammar, these expectations can be used as constraints on possible constituent structures of a HPSG constituent. Given this mapping, the expectations may then be used to order the parse process, guiding the parse, and avoiding the consideration of pragmatically unlikely constructions. This process reduces the number of constituents considered during parsing, reducing parse time and permitting the parser to correctly select the parse most like the pragmatic expectations.

This solution closely follows a classical A.I. search technique called a best-first search. The heuristic evaluation function used to classify the proposed constituents for best first ordering uses inference similar to abductive reasoning.

One benefit of this solution is that it retains the modularity of the syntactic and semantic components, not requiring a specialized grammar for each contextual domain. In additional, as the coverage of the grammar increases, the search space will also increase, and thus possible benefits increase.

Work is continuing on this study. Currently the heuristic is being enhanced to consider the specificity of an expectation match, ordering those edges which match the most specific features first. In addition, work is in progress to extend the coverage of the grammar and mapping to include the conversation domain utilized in Patten, Geis \& Becker 1992.

\section{References}

Geis, Mike. L. and Harlow, L. "Politeness Strategies in French and English: Implications for Second Language Acquisition"

Mac Gregor, R., "LOOM Users Manual", University of Southern California, Informations Sciences Institute, 1991.

Patten, Terry.; Geis, Mike. and Becker, Barbara., "Toward a Theory of Compilation for Natural-Language Generation," Computational Intelligence 8(1), 1992, pp 77-101.

Pollard, Carl and Sag, Ivan A., "Head-Driven Phrase Structure Grammar", unpublished manuscript draft, 1992.

Pollard, Carl. and Sag, Ivan A., "Information-Based Syntax and Semantics: Volume 1, Fundamentals", Center for the Study of Language and Information, 1987.

Schegloff, E.A. and Sacks, H. Opening up closings. Semiotica, 7,4:289-387, 1973.

Winograd, Terry. 1983. "Language as a Cognitive Process", Addison-Wesley, Menlo Park, CA. 